\title{
Characteristics of Water in Polypeptide Membrane
}

\author{
Takatoshi KInoshitA, Tetsuya TANigAmi, Shintaroh WASHIzU, \\ Sadanobu HAYASHI, Akira TAKIZAWA, \\ and Yoshiharu TSUJITA \\ Department of Fiber and Polymer Engineering, Nagoya Institute of Technology, \\ Gokiso-cho, Showa-ku, Nagoya 466, Japan.
}

(Received December 12, 1979)

\begin{abstract}
In order to elucidate the character of water in polypeptide membranes, sorption, and diffusion of water vapor were measured in membranes of poly(L-leucine) $(\mathbf{I})$ and poly $(\gamma$-methyl L-glutamate) (II). Water sorption in $\mathbf{I}$ is about $1 / 5$ of that in $\mathbf{I I}$ in a high relative vapor-pressure region and is about a hundred-fold greater than in polyolefines. The apparent diffusion coefficient of the system of water vapor and $\mathbf{I}$ at low temperature at a relative vapor pressure $p / p_{0}=0.8$ is lower than at $p / p_{\mathrm{o}}=0.2$. This is due to the clustering tendency of water molecules in $\mathbf{I}$. At a low temperature, the diffusion coefficients of the systems of ions and $\mathbf{I}$ are smaller than those of ions and II. This is also attributable to water clustering in I. Shear moduli of water-containing I and II membranes were measured by the free-damping oscillation method at certain fixed relative vapor pressures. Though the shear modulus of I is lower than that of II in the dry state, the increase of the former with increasing water content is remarkable, and exceeds the value for II by a volume fraction of water of about 0.1 . This also reflects a rigid water structure due to clustering in $\mathbf{I}$.

KEY WORDS Poly(L-leucine) / Poly $(\gamma$-methyl L-glutamate) / Sorption / Diffusion Coefficient / Shear Modulus / Water Clustering / Rigid Water Structure /
\end{abstract}

It has been shown that the permeabilities of poly(L-leucine) (PLL) and poly( $\gamma$-methyl L-glutamate) (PMLG) membranes to water are of the same order, but that PLL membranes are more efficient than PMLG membranes for separating watersoluble solutes. ${ }^{1}$ It is supposed that the dissolution of water-soluble solute in the PLL membrane is poor because of the hydrophobic nature of the side chain in spite of the rather high diffusivity of water in the membrane. Also, it seems that PLL membranes become somewhat rigid when exposed to water. From these observations, it may be inferred that water in PLL forms rigid clusters which cannot behave like free water. Although the clustering tendency of water in hydrophobic polymers has been reported in many cases, ${ }^{2}$ the effect of this on membrane characteristics is not clear, probably because the usual hydrophobic polymer sorbs too little water to show a change in the membrane characteristics. Since the sorption of water in various polypeptides takes place in the rather large side-chain region between helices, ${ }^{3}$ the amount sorbed is expected to be greater than that in ordinary hydrophobic polymers. In this paper, in order to elucidate the behavior of water toward a membrane in regard to the characteristics of the membrane, the sorption and diffusion of water vapor at various relative vapor pressures and temperatures, ionic permeabilities at various temperatures, and the shear moduli of water-containing membranes at various relative vapor pressures are examined using the PLL membrane, and a comparison in these respects with the more hydrophilic PMLG membrane is made.

\section{EXPERIMENTAL}

\section{Materials}

Poly(L-leucine) (PLL) was prepared by the polymerization of L-leucine $N$-carboxyanhydride (NCA) by triethylamine as an initiator in benzene for one week at $30^{\circ} \mathrm{C}$. The viscosity-average molecular weight using trifluoroacetic acid solution is $2.7 \times 10^{4}$. The PLL membrane was prepared by 
casting on the glass plate at $50^{\circ} \mathrm{C}$, pouring the $1 \%$ benzene solution at $70^{\circ} \mathrm{C}$. After the solvent was removed, the residual solvent was substituted by methyl alcohol, and the membrane thus formed was dried and annealed at $80^{\circ} \mathrm{C}$ for 24 hours under vacuum. The thickness of the membrane was about $2 \times 10^{-2} \mathrm{~cm}$. The density of the PLL membrane observed by the floatation method using toluenecarbon tetrachloride solution was $0.990 \mathrm{~g} \mathrm{~cm}^{-3}$. Poly $(\gamma$-methyl L-glutamate) (PMLG) was supplied by Kyowa Hakko Kogyo Co. Ltd. The molecular weight was about $1.5 \times 10^{5}$. The PMLG membrane was prepared by the same method as that of PLL except that the original cast solution was $5 \%$ ethylene dichloride solution. The thickness of the membrane was also $2.0 \times 10^{-2} \mathrm{~cm}$. The density of the membrane was $1.32 \mathrm{~g} \mathrm{~cm}^{-3}$. Both the PLL and the PMLG membranes had the $\alpha$-helical structure, as confirmed by infrared adsorption spectra. The $\mathrm{KCl}$ used for the ionic-permeability measurement was of extra pure grade.

\section{Sorption and Permeation of Water Vapor}

Sorption and permeation were studied mainly for PLL in this study. Sorption experiments were made by a gravimetric sorption apparatus using a quartz spring balance. ${ }^{4}$ Sorption isotherms were expressed as the relation between the amount of sorbed water $\mathrm{mol} /(\mathrm{g}$ of residue), $n$, and relative water vapor pressure $p / p_{0}$. The isotherms were observed at 10 , 25 , and $40^{\circ} \mathrm{C}$. For permeation experiments, the Rouse-type apparatus was used. ${ }^{5}$ On the feed-side or high-pressure side of the membrane, water vapor at a controlled pressure was supplied, by the temperature regulation of water in a trap connected to the cell. The pressure on the permeant-side or lowpressure side cell was essentially zero, and liquid nitrogen was used to trap the permeant vapor. From the slope of the steady-state straight-line portion of the permeation curve (amount permeated $v s$. time relation) the permeability coefficient $\bar{P}\left(\mathrm{~cm}^{3} \mathrm{stp}\right.$ $\mathrm{cm}^{-1} \mathrm{~s}^{-1} \mathrm{cmHg}^{-1}$, the bar denotes concentration average.) was calculated as usual. Since the sorption isotherm does not obey Henry's law, the "apparent" average diffusion coefficient $\bar{D}\left(\mathrm{~cm}^{2} \mathrm{~s}^{-1}\right)$ was obtained by $\bar{P} / S$, where $S$ is the solubility coefficient.

\section{Ionic Permeability}

The measurement of the permeability of ions through the membranes was carried out in a manner analogious to that of Tasaka. ${ }^{6}$ The whole apparatus was made by Acrylate resin (Mitsubishi Rayon Co. Ltd., Japan). The membrane was set up with Teflon plate support and silicon rubber packing on the permeation cell. The effective permeation area was $7.07 \mathrm{~cm}^{2}$. The feed-side compartment of the cell was filled with an aqueous solution of $\mathrm{KCl}$ of initial concentration, $C_{1} \mathrm{moll}^{-1}\left(5 \times 10^{-3} \mathrm{moll}^{-1}\right.$ for PLL and $10^{-1} \sim 2 \times 10^{-3} \mathrm{moll}^{-1}$ for PMLG). The salt concentration of the permeated-side compartment was $C_{2} \mathrm{moll}^{-1}\left(5 \times 10^{-4} \mathrm{moll}^{-1}\right.$ for PLL and $1.25 \times 10^{-2} \sim 2 \times 10^{-4} \mathrm{moll}^{-1}$ for PMLG). The change in the concentration with time was measured with an electroconductivity-meter (Toa Electronics, Ltd., Japan, CN-5B Type). The changes in both the feed and permeant concentration with time were small compared with the initial concentrations. The whole cell was immersed in a water bath whose temperature was controlled to within $0.1^{\circ} \mathrm{C}$. The measurement of ionic permeability was carried out at temperatures between $10 \sim 35^{\circ} \mathrm{C}$ at $5^{\circ} \mathrm{C}$ intervals. From the steady-state straight line of the relation between conductance of the permeant and time, the flux of $J_{\mathrm{s}}$ (which is equal to the flux of ion in the case of $\mathrm{KCl}$ ) was calculated. Then, using eq 1 , permeability of salt or ions $P_{\mathrm{s}}\left(\mathrm{cm}^{2} \mathrm{~s}^{-1}\right)$ was obtained,

$$
P_{\mathrm{s}}=J_{\mathrm{s}} \Delta x /\left(C_{1}-C_{2}\right)
$$

where, $\Delta x$ is the membrane thickness. The diffusion coefficient of the system $D_{\mathrm{s}}\left(\mathrm{cm}^{2} \mathrm{~s}^{-1}\right)$ was obtained by $P_{\mathrm{s}} / K_{\mathrm{s}}$, where $K_{\mathrm{s}}$ is the solubility coefficient of salt. In order to obtain $K_{\mathrm{s}}$, about $0.08 \mathrm{~g}$ of the membrane was immersed in a salt solution of desired concentration, and after the sorption equilibrium was attained, the membrane was removed, rinsed, blotted, and immersed in distilled water. From the value of conductance of the solution at desorption equilibrium, the amount of salt $\bar{C}$ (moll ${ }^{-1}$ in swollen membrane), which had been present in the membrane, was calculated. The $K_{\mathrm{s}}$ is defined as the ratio of $\bar{C}$ to $C$ ( $\mathrm{moll}^{-1}$ on initial solution). In this case, $K_{\mathrm{s}}$ is independent of concentration and temperature. ${ }^{13}$

\section{Shear Modulus of Water-Containing Membrane}

In order to obtain the shear modulus of a membrane in which a known amount of water is sorbed, we made the observation for the membrane in a closed system (Figure 1). The usual free oscillating pendulum, consisting a sample mem- 


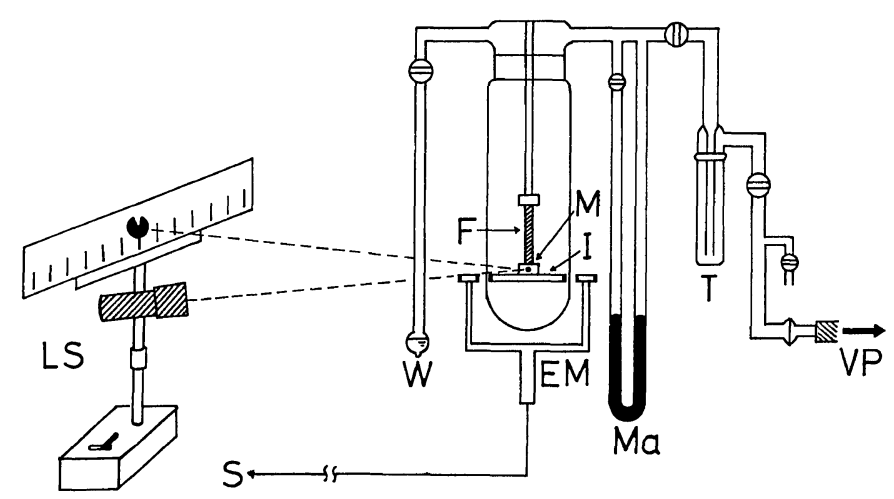

Figure 1. Schematic diagram of tortion pendulum in a closed system: F, sample membrane; M, mirror; I, inertia rod; EM, electromagnet; W, water flask; Ma, manometer; LS, lamp scale; T, trap; S, switch; VP, vacuum pump.

brane $\mathrm{F}$, mirror $\mathrm{M}$ and inertia rod $\mathrm{I}$ on both ends of which iron nuts is attached, was set up in an airtight chamber system. Following the evacuation of the whole system at $25^{\circ} \mathrm{C}$ for 3 to 4 days, water vapor was introduced into the system successively by controlling the temperature of water-supply flask W. The relative water-vapor pressure thus introduced was observed by the manometer Ma. The sorption equiliblium was attained in two days, as could be judged by the pressure change in the manometer. To induce the oscillation, the electromagnet EM was excited instantaneously to produce the initial torsional angle of $10^{\circ}$. After the initiation of the oscillation, the period and amplitude of the free damping oscillation were measured by the lamp scale LS, as usual. The frequency in this case was about $0.22 \mathrm{~Hz}$. The relative modulus $G_{\mathrm{r}}$, which is a measure of the change of rigidity, is defined as the ratio of the modulus under a certain relative vapor pressure $G_{\mathrm{i}}$ to that in the dry state $G_{\mathrm{o}}$, and is obtained by eq 2 ,

$$
G_{\mathrm{r}}=\left(G_{\mathrm{i}} / G_{\mathrm{o}}\right)=\left(T_{\mathrm{o}} / T_{\mathrm{i}}\right)^{2}
$$

where, $T_{\mathrm{o}}$ and $T_{\mathrm{i}}$ are periods of oscillation in the respective cases. The logarithmic decrement $\Delta$ is calculated by the average of the logarithm of the ratio of amplitudes over one cycle (eq 3 ), and is related to the energy loss resulting from free damping oscillation. ${ }^{7}$

$$
\begin{aligned}
\Delta & =\ln \left(A_{0} / A_{1}\right)=\ln \left(A_{1} / A_{2}\right) \\
& =\cdots=\ln \left(A_{n-1} / A_{n}\right) \\
& =\text { const }
\end{aligned}
$$

where the subscript $n$ on the amplitude $A$ denotes the number of the cycle. The absolute value of a shear modulus $G_{0}$ was obtained by a torsional free oscillating rheometer (Ohyo Denki Kenkyusho, Co. Ltd., Japan, RD-1 Type), employing dry samples of PLL and PMLG membranes in evacuated state $\left(10^{-2} \mathrm{mmHg}\right) . G_{0}$ is calculated by eq 4 ,

$$
G_{\mathrm{o}}=L\left\{\frac{\left(4 \pi^{2}+\Delta^{2}\right) I}{T^{2}}-k_{\mathrm{o}}\right\}
$$

where, $I$. is the moment of inertia and $k_{\mathrm{o}}$ is the torsion constant of the wire. The values used in this study were $I=1.33 \times 10^{3} \mathrm{~g} \mathrm{~cm}^{-2}, k_{\mathrm{o}}=0.734 \times 10^{2}$ dyn $\mathrm{cm}^{-1}$. $L$ is the shape factor represented by eq 5 .

$$
L=\frac{l}{\beta b c^{3}}
$$

where $l$ is the sample length, $5 \mathrm{~cm}$, and $b$ is the sample width, $1 \mathrm{~cm}$, and $c$ is the thickness, $0.008 \mathrm{~cm}$. $\beta[=(1-0.63 c / d) / 3]$ is the constant, being $1 / 3$, in this case.

\section{RESULTS AND DISCUSSION}

\section{Sorption and Permeation of Water Vapor}

Sorption isotherms of water in PLL at various temperatures are shown in Figure 2. The effect of the hydrophobic nature of PLL is also shown in this figure. The amount of water in PLL is less than 0.1 mol per residue and is about $1 / 5$ of that in PMLG (dotted line in Figure 2) at high relative pressure at $25^{\circ} \mathrm{C}$. However, water sorption in PLL is a hundred- 


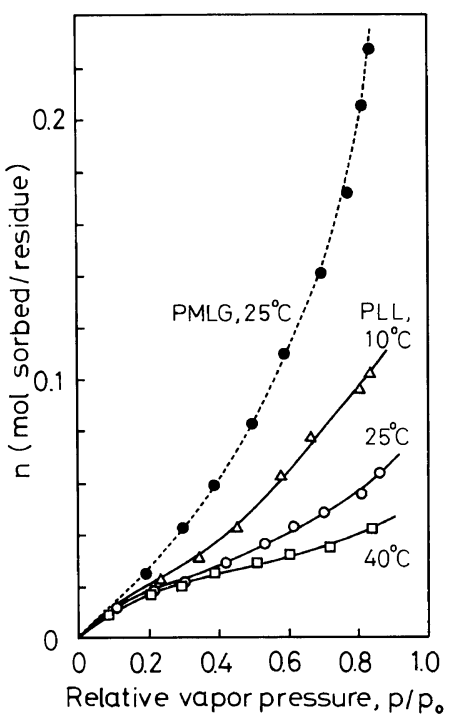

Figure 2. Sorption isotherms of water by $\operatorname{PLL}(10,25$, and $40^{\circ} \mathrm{C}$ and by PMLG $\left(25^{\circ} \mathrm{C}\right)$.

fold greater than that in polyolefines, if comparison is made in the high relative vapor-pressure range. The difference between water solubility in PLL and that in polyolefines is due to the relatively large inter-helix regions of PLL. In strong contrast to the isotherms of ordinary hydrophobic polymer-water systems, those of the PLL-water system depend remarkably on temperature. The isotherms of the PMLG-water system were almost independent of temperature. ${ }^{9}$ Ordinary hydrophilic polymer shows large temperature dependence on water sorption at low relative vapor pressure; but on the other hand, the water-sorption isotherms of PLL at different temperatures deviate from each other at high relative pressures. This result shows that the heat of sorption increases with increasing vapor pressure; that is, water in PLL forms a structure more compact than the liquid-like structure in the high vapor-pressure range. To make clear the size of water cluster in membrane, the concept of cluster function defined by Zimm-Lundberg ${ }^{10}$ was applied to the present systems,

$$
G_{11} / V_{1}=-V_{2}\left[\frac{\partial\left(a_{1} / v_{1}\right)}{\partial a_{1}}\right]_{p, \mathrm{~T}}-1
$$

where $v_{1}$ and $v_{2}$ are the volume fractions of water and polymer, respectively, $V_{1}$ is the molecular volume of water, $a_{1}$ is the activity of water vapor,

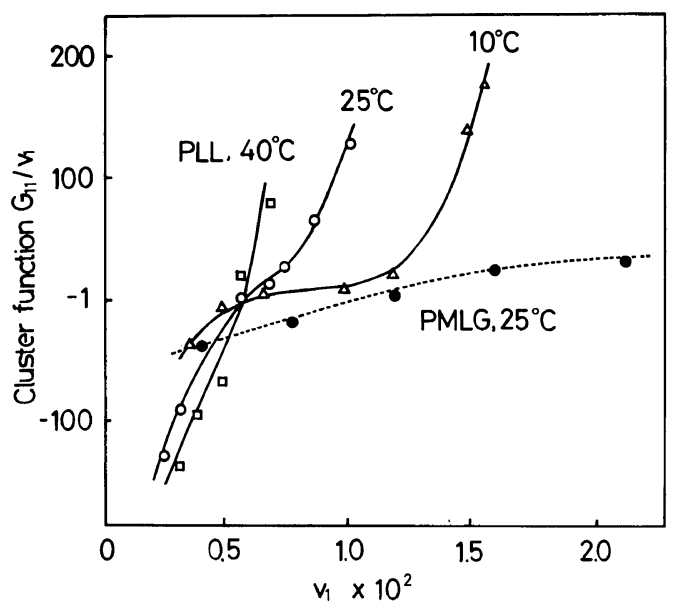

Figure 3. Cluster function of water in PLL $(10,25$, and $\left.40^{\circ} \mathrm{C}\right)$ and in PMLG $\left(25^{\circ} \mathrm{C}\right)$.

which is approximated by the relative vapor pressure $p / p_{0}$, and $G_{11}$ is the cluster integral. The calculation result is shown in Figure 3 as a plot of the cluster function against the volume fraction of water in the membrane. Since the present system is composed of helical rods, a rubbery side-chain region and water, the values of Figure 3 may indicate the clustering tendency, and the absolute values should be considered with caution. At all temperatures, the cluster function shows that the water in PLL is segregated below $v_{1} \simeq 0.006$, where the $G_{11} / V_{1} \simeq-1$. After some sites have been filled up (corresponding $v_{1}$ is independent on temperature), the clustering tendency becomes remarkable. This rapid increase in the cluster function is significant compared with the case of relatively hydrophobic polymers, e.g., poly(vinyl acetate $)^{11}$ and poly(dimethylsiloxane). ${ }^{12}$ The cluster function in the case of PMLG $\left(25^{\circ} \mathrm{C}\right)^{9}$ is clearly lower than that of PLL at the same volume fraction of water in the polymer.

The steady-state permeability $\bar{P}$ of water vapor through the PLL membrane vs. the relative vapor pressure $p / p_{0}$ at various temperatures is shown in Figure 4 , together with the steady-state diffusion coefficient $\bar{D}$. Both the $\bar{P}$ and $\bar{D}$ increase with temperature, but the dependency of $\bar{D}$ on relative vapor pressure is somewhat complex. $\bar{D}$ passes through a maximum as the relative vapor pressure increases, and the vapor pressure corresponding to the maximum of $\bar{D}$ shifts to the high relative vapor- 


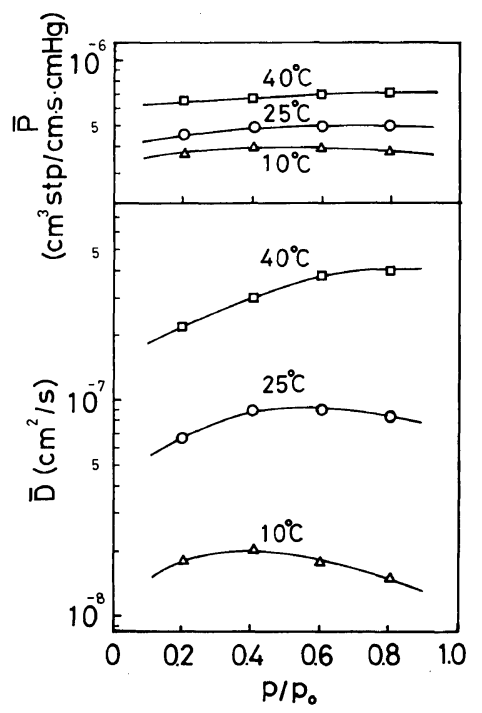

Figure 4. Steady-state permeability coefficient $\bar{P}$ and diffusion coefficient $\bar{D}$ of water in PLL at 10, 25, and $40^{\circ} \mathrm{C} v s$. relative vapor pressure $p / p_{\text {o }}$ of water.

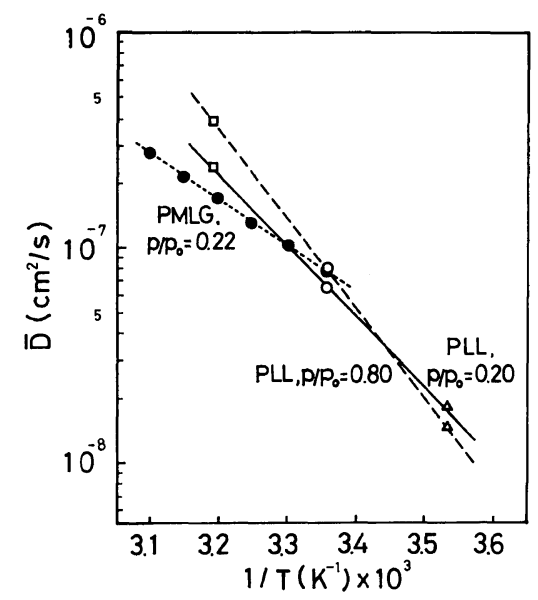

Figure 5. Arrhenius plot of the diffusion coefficient of water in PLL (solid line represents the case when relative vapor pressure $p / p_{\mathrm{o}}$ is held to 0.20 at each temperature, broken line represents the case in which $p / p_{\mathrm{o}}$ is held to 0.8 ) and PMLG (dotted line, $p / p_{\mathrm{o}}$ is held to 0.22 ).

pressure side as the temperature rises. This may be ascribed to the following two factors: the plasticizing effect of segregated water and the clustering tendency of water, shown in Figure 3. To make a clear assessment of these respective effects, the values of $\bar{D}$ at each temperature were evaluated at fixed relative vapor pressures, 0.2 and 0.8 . Figure 5 shows the results in the Arrhenius plot. At higher temperatures, $\bar{D}$ at $p / p_{\mathrm{o}}=0.8$ is larger than that of $p / p_{\mathrm{o}}=0.2$. This suggests that the plasticizing effect of water is rather prominent in this temperature region. At lower temperatures, the clustering tendency and the change in structure of water reduce $\bar{D}$ at high relative pressure. This structural change from free water was comfirmed by preliminary IR and broad-line NMR measurements. The details for this will be given elsewhere. The activation energies at 0.2 and 0.8 of $p / p_{\mathrm{o}}$, are 15.3 and $19.1 \mathrm{kcal} \mathrm{mol}^{-1}$, respectively. For the sake of comparison, the value of $\bar{D}$ of the water-PMLG system, evaluated at $p / p_{\mathrm{o}}=0.22,{ }^{1}$ is also shown in the figure. In the latter system, the effect of structural change in water with temperature is considered to be minor, and thus the activation energy of this system, $10.3 \mathrm{kcal} \mathrm{mol}^{-1}$, is lower than that of PLL-water system.

\section{Ionic Permeability}

Figure 6 shows the permeability coefficient $P_{\mathrm{s}}$ of $\mathrm{KCl}$ through $\mathrm{PLL}$ against temperature, in the

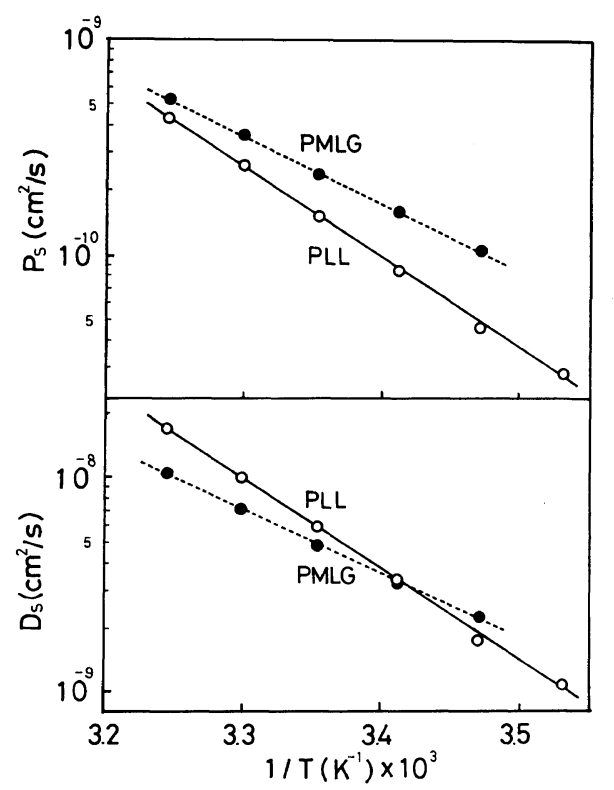

Figure 6. Arrhenius plot of permeability coefficient $P_{s}$ and diffusion coefficient $D_{\mathrm{s}}$ of $\mathrm{KCl}$ in PLL (solid line) and PMLG (broken line) membrane. 
Arrhenius plot. The data of PMLG ${ }^{13}$ is also given in the figure. It has been shown that $P_{\mathrm{s}}$ does not depend on either $C_{1}$ or $C_{1} / C_{2}$ in the present concentration range, and so $P_{\mathrm{s}}$ may be considered to be constant. ${ }^{13}$ The activation energies of permeation for PLL and PMLG are obtained as 19 and $14 \mathrm{kcal}$ $\mathrm{mol}^{-1}$, respectively. The solubility coefficients $K_{\mathrm{s}}$ of $\mathrm{KCl}$ in PLL and PMLG are obtained as $2.5 \times 10^{-2}$ and $5.2 \times 10^{-2}$, respectively, and these values are independent of ionic concentration and temperature. ${ }^{13}$ Using these values, $D_{\mathrm{s}}$ is calculated, and is shown in Figure 6 . Though in the high temperature region, $D_{\mathrm{s}}$ in PLL is higher than that in PMLG, at low temperatures this relation is reversed. Since the amount of water in the membranes remains constant over the temperature range studied, this behavior may be explained by the structural change of water with temperature in the membrane. Particularly in the PLL membrane, it is considered that water makes relatively rigid clusters in the lowtemperature range, and that these clusters reduce the diffusivity of ion in the membrane.

From the results of water-vapor sorption and diffusion, and ionic permeability in PLL membrane, it is concluded that the hydrophobic nature of the PLL side chain not only reduces the water content compared with that of PMLG membrane, but also facillitates the clustering tendency of water which leads to a decrease in solute solubility in PLL because of the rigid - an ice-like-structure. This slight solubility of solute may be the main reason for the excellent separation of water-soluble solute through PLL membrane. ${ }^{1}$

\section{Shear Modulus of Water-Containing Polypeptide Membrane}

The structure of water in PLL may be confirmed by the mechanical behavior of water-containing polypeptide membranes. In Figure 7, the relative modulus $G_{\mathrm{r}}$ and the logarithmic decrement $\Delta$ of the PLL-water system are plotted against the relative water-vapor pressure $p / p_{\mathbf{o}}$. The sorption isotherm, which is the volume fraction of water $v_{1} v s . p / p_{\mathrm{o}}$ relation, is also shown in the figure. Though the modulus increases with $v_{1}$ gradually up to $v_{1} \simeq 0.004$, a further increase with $v_{1}$ is significant, and this may indicate that a cluster of water molecules makes a relatively rigid structure in PLL. Namely, the water cluster may carry out the role of a filler ${ }^{14}$ in regard to the modulus. The $\Delta$ does not

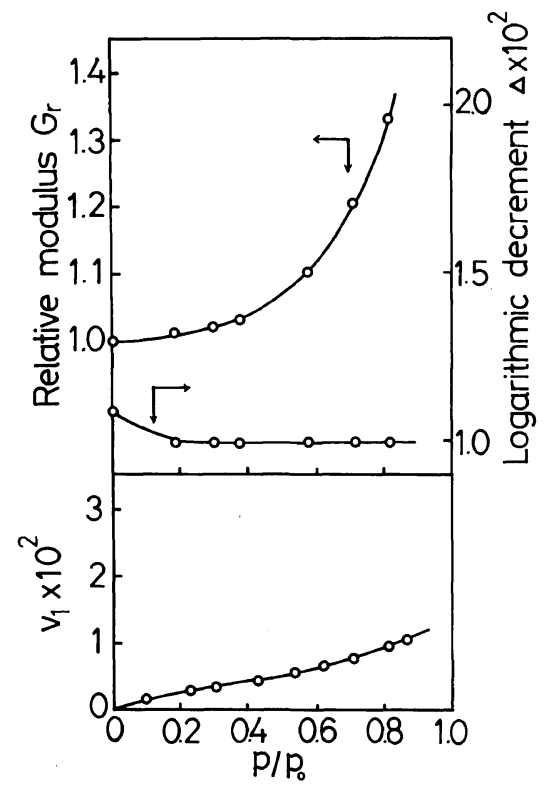

Figure 7. Relative modulus $G_{\mathrm{r}}$ and logarithmic decrement $\Delta$ of water-contained PLL membrane $v s$. relative vapor pressure $p / p_{\mathrm{o}}$. The isotherm is expressed by the relation between volume fraction of water in the membrane $v_{1}$ and $p / p_{0}$.

change appreciably, and this shows that the frictional energy loss in the side-chain region of PLL is not affected by adsorbed water molecules. The results for PMLG membrane are shown in Figure 8. $G_{\mathrm{r}}$ decreases once near $p / p_{\mathrm{o}}=0.1$. The sorption of water molecules at its initial stage may occur on the relatively polar carboxyl group of the side chain, and enhances the molecular movement of the side chain by the plascizing effect. However, since the other part of side chain of PMLG is hydrophobic, with a further increase of water in the membrane, the same behavior as that of the PLL membrane is observed. The decrease of $\Delta$ shows that the sample becomes elastic by an interaction between side chain and water that is stronger than that in the PLL membrane. The absolute moduli of the dry samples of PLL and PMLG membranes, described in the experimental section, were obtained as $G_{0}=1.89 \times 10^{10} \quad \mathrm{dyn} \mathrm{cm}^{-2}$ for PLL and $G_{\mathrm{o}}=2.41 \times 10^{10} \mathrm{dyn}^{-2}$ for PMLG, respectively. Using these values, in Figure 9, the absolute modulus $G$ is plotted against the volume fraction of water $v_{1}$ in the membrane for both PLL and PMLG membranes. In spite of the high modulus of PMLG 


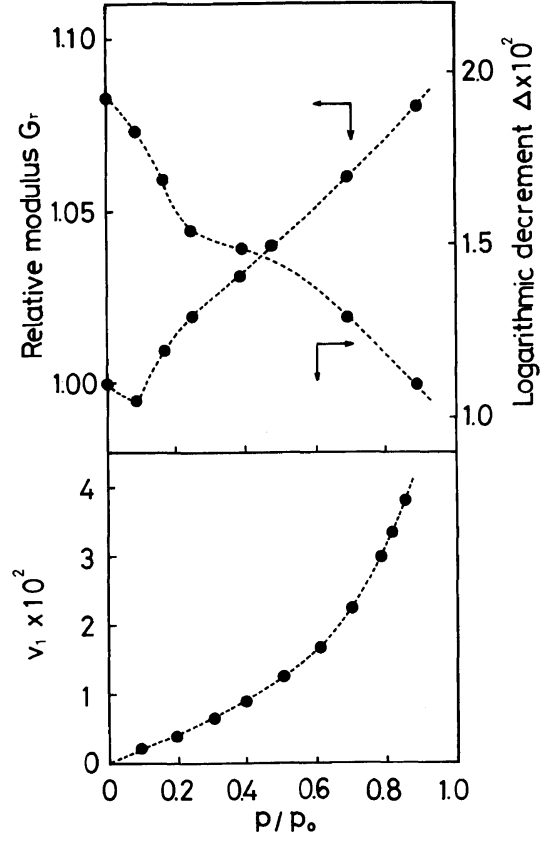

Figure 8. Relative modulus $G_{\mathrm{r}}$ and logarithmic decrement $\Delta$ of water-contained PMLG membrane $v s$. relative vapor pressure $p / p_{0}$. The isotherm is expressed by the relation between volume fraction of water in the membrane $v_{1}$ and $p / p_{0}$.

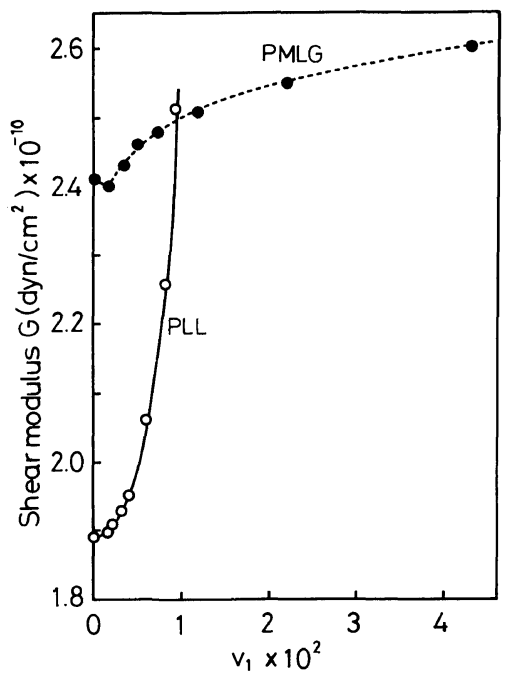

Figure 9. Absolute value of shear modulus $G v s$. volume fraction of water $v_{1}$ for water-contained PLL and PMLG membranes. membrane in the dry state due to the interaction among side chains, the increase in $G$ with $v_{1}$ for PLL is remarkable compared with that of PMLG, and the relations for both membranes intersect at about $v_{1}=0.009$. This behavior agrees well with the rigidity of the PLL membrane in the water and with the various transport phenomena described above, and also gives direct evidence for the rigid structure of water in PLL membrane.

Acknowledgement. The authors gratefully acknowledge the support of the Ministry of Education, Science and Culture, Japan for the Grant-in-Aid for Scientific Research (No. 321117) in 1978. We also express our appreciation to Kyowa Hakko Kogyo Co. Ltd. for supplying the samples.

\section{REFERENCES}

1. M . Maeda, K. Yokoi, A. Takizawa, and Y. Tsujita, Kobunshi Ronbunshu, 34, 779 (1977); T. Taniguchi, I. Yamamuro, A. Takizawa, and Y. Tsujita, $J$. Macromol. Sci., Phys., B17, 203 (1977).

2. J. A. Barrie, "Diffusion in Polymers," J. Crank and G. S. Park, Ed., Academic Press, London, New York, N.Y., 1968, Chapter 8.

3. T. Hamada and A. Takizawa, Polymer, 15, 157 (1974); T. Hamada and A. Takizawa, J. Appl. Polym. Sci., 18, 1443 (1974); T. Taniguchi, I. Yamamuro, A. Takizawa, and Y. Tsujita, J. Macromol. Sci., Phys., B13, 203 (1977).

4. A. Takizawa, J. Polym. Sci., 6, 476 (1968).

5. R. E. Rouse, J. Am. Chem. Soc., 60, 1068 (1947).

6. M. Tasaka, Y. Kondo, and M. Nagasawa, J. Phys. Chem., 73, 3181 (1969).

7. The Society of Polymer Science, Japan, Ed., "Polymer Characterization, Structure, and Properties," I, (in Japanese), Baifukan, Tokyo 1973.

8. The Society of Polymer Science, Japan, Ed., "Polymer and Water," (in Japanese), Saiwai-shyobo, Tokyo, 1972, Chapter 5.

9. T. Hamada, J. Shiomokawa, and A. Takizawa, Kobunshi Kagaku, 28, 751 (1971).

10. B. H. Zimm and J. L. Lundberg, J. Phys. Chem., 60, 425 (1956); J. L. Lundberg, Pure Appl. Chem., 31, 261 (1972).

11. H. W. Starkweather, J. Polym. Sci., B, 1, 133 (1963).

12. J. A. Barrie and B. Platt, Polymer, 4, 303 (1963).

13. T. Kinoshita, S. Hayashi, A. Takizawa, and Y. Tsujita, J. Macromol. Sci., Phys., B17, 39 (1980).

14. F. Bueche, "Physical Properties of Polymers," Interscience Publishers, New York N.Y., London, 1962, Chapter 2. 